\title{
Quantum phenomena in the radial thermal expansion of bundles of single-walled carbon nanotubes doped with ${ }^{3} \mathrm{He}$. A giant isotope effect
}

\author{
A. V. Dolbin $*$ V. B. Esel'son, V. G. Gavrilko, V. G. Manzhelii, N. A. Vinnikov, and S. N. Popov \\ B. Verkin Institute for Low Temperature Physics 83 Engineering NASU, Kharkov 61103, Ukraine \\ B. Sudqvist \\ Department of Physics, Umea University, SE-901 87 Umea, Sweden
}

(Dated: December 5, 2018)

\begin{abstract}
The radial thermal expansion $\alpha_{r}$ of bundles of single-walled carbon nanotubes saturated with ${ }^{3} \mathrm{He}$ up to the molar concentration $9.4 \%$ has been investigated in the temperature interval $2.1-9.5 \mathrm{~K}$ by high-sensitivity capacitance dilatometry. In the interval $2.1-7 \mathrm{~K}$ a negative $\alpha_{r}$ was observed, with a magnitude which exceeded the largest negative $\alpha_{r}$ values of pure and ${ }^{4} \mathrm{He}$-saturated nanotubes by three and two orders of magnitude, respectively. The contributions of the two He isotope impurities to the negative thermal expansion of the nanotube bundles are most likely connected with the spatial redistribution of ${ }^{4} \mathrm{He}$ and ${ }^{3} \mathrm{He}$ atoms by tunneling at the surface and inside nanotube bundles. The isotope effect turned out to be huge, probably owing to the higher tunneling probability of ${ }^{3} \mathrm{He}$ atoms.

PACS numbers: 65.60.+a, 65.80.-g, 65.40.De, 64.70.Tg
\end{abstract}

\section{INTRODUCTION}

Since their discovery by Iijima in $1991^{1}$, carbon nanotubes (CNTs) have been attracting intense interest from scientists owing to their unique geometry and extraordinary physical properties. The very high length-todiameter ratios and the capability of CNTs to form bundles of several tens or even hundreds of tubes make it possible to form low-dimensional, ordered impurity phases at the bundles' surfaces ${ }^{2.3}$. Such phases consist of impurity molecules or atoms forming one-dimensional chains in the intertube grooves or in the interstitial channels in the bundles. They can also form two-dimensional layers at the bundle surface. It has been found experimentally $\underline{\underline{4}} \underline{\underline{7}}$ that the radial thermal expansion coefficients $\alpha_{r}$ of nanotube bundles are negative in the region of liquid helium temperatures. Gas impurities usually suppress the magnitudes of these negative values of $\alpha_{r}$ and reduce the temperature region where they exist. The ${ }^{4} \mathrm{He}$ impurity is an exception: when ${ }^{4} \mathrm{He}$ is introduced both the magnitude of the negative $\alpha_{r}$ values and the temperature region of the negative thermal expansion increase ${ }^{7}$. This effect was attributed to a tunneling redistribution of the ${ }^{4} \mathrm{He}$ atoms at the surface and inside CNT bundles. It is known ${ }^{8,9}$ that the processes of tunneling gives a negative contribution to the thermal expansion of a system. It is then reasonable to expect that saturation of CNT bundles with ${ }^{3} \mathrm{He}$ would enhance the above effect because the smaller masses of ${ }^{3} \mathrm{He}$ atoms must increase the probability of tunneling.

In the present work we have, therefore, investigated the radial thermal expansion of single-walled carbon nanotubes (SWNTs) saturated with ${ }^{3} \mathrm{He}$ using the dilatometric method. The temperature interval studied was 2.1$9.5 \mathrm{~K}$. As will be shown below, the experimental results verify our expectation that the addition of ${ }^{3} \mathrm{He}$ should enhance the negative thermal expansion; in fact the ef- fect is surprisingly large, two orders of magnitude larger than for ${ }^{4} \mathrm{He}$.

\section{EXPERIMENTAL TECHNIQUE}

The radial thermal expansion of ${ }^{3} \mathrm{He}$ saturated CNTs was investigated using a high-sensitivity low-temperature capacitance dilatometer with $2 \cdot 10^{-9} \mathrm{~cm}$ resolution. The technique and the experimental apparatus are presented in detail elsewhere $\stackrel{10}{\underline{10}}$. The sample was a cylinder $7.2 \mathrm{~mm}$ high and $10 \mathrm{~mm}$ in diameter, obtained by compressing a stack of thin $(\leq 0.4 \mathrm{~mm})$ plates consisting of in-plane oriented CNTs at $1.1 \mathrm{GPa}$. The plates were prepared by compressing (1.1 GPa) small amounts of CNT powder (Cheap Tubes, USA, CCVD method). It is known 11 that such pressure treatment of a thin CNT layer leads to a preferred orientation where the CNT axes mainly lie in the plane perpendicular to the applied pressure, the average deviation being about $4^{\circ}$. The alignment of CNT axes in the plane makes it possible to investigate preferentially the radial component of the thermal expansion of the tubes $\frac{12}{2}$ and the effect of gas saturation upon the radial thermal expansion of SWNT bundles ${ }^{4-7}$.

Just before starting the investigation, the cell with a pure CNT sample was evacuated at room temperature for 72 hours to remove possible gas impurities. It was then cooled to $2.1 \mathrm{~K}$ and a series of control measurements was performed. The results showed that the thermal expansion of the sample coincided, within the experimental error, with the values obtained previously for pure $\mathrm{CNTs}_{\mathrm{s}} 12$ (see Fig. 11a, curve 4). ${ }^{3} \mathrm{He}$ gas was then fed to the measuring cell at $\mathrm{T}=2.1 \mathrm{~K}$. The ${ }^{3} \mathrm{He}$ was added in small portions as some quantities were sorbed by the nanotubes. This permitted us to maintain the pressure in the cell several times lower than the pressure of saturated ${ }^{3} \mathrm{He}$ vapor at this temperature (151.112 Torr at 
$\left.\mathrm{T}=2 \mathrm{~K}^{13}\right)$. The total amount of ${ }^{3} \mathrm{He}$ absorbed by the pure CNT sample was $9.4 \mathrm{~mol}$. \% $\left(94{ }^{3} \mathrm{He}\right.$ atoms per $1000 \mathrm{C}$ atoms). At this impurity concentration we were able to compare our results on the thermal expansion of the ${ }^{3} \mathrm{He}-\mathrm{SWNT}$ with those from previous measurements of the radial thermal expansion of CNTs saturated with ${ }^{4} \mathrm{He}$ to the molar concentration $9.4 \%{ }^{\underline{7}}$. After the sorption was completed, an equilibrium of $\sim 1 \cdot 10^{-4}$ Torr was set in the measuring cell. Since the rise of the sample temperature in the course of measuring $\alpha_{r}$ could entail some ${ }^{3} \mathrm{He}$ desorption from the sample, the reproducibility of the results was checked at regular intervals by heating and subsequently cooling the sample by $\Delta T$, where $\Delta T=0.3 \ldots 1 \mathrm{~K}$. If the results obtained under this cycling coincided, within experimental error, the effect of He desorption was regarded as negligible and the data were considered to be obtained in equilibrium. The absence of reproducibility was believed to show that at this and higher temperatures the desorption of the ${ }^{3} \mathrm{He}$ impurity from the sample had some effect on the thermal expansion. The measurement was then stopped. Note that for the radial thermal expansion of the ${ }^{3} \mathrm{He}-\mathrm{SWNT}$ the data were observed to be reproducible in the interval $\mathrm{T}=2.1-9.5 \mathrm{~K}$, but no longer reproducible when cooling the sample to $9.7 \mathrm{~K}$. When reproducibility was no longer observed the sample was heated to $\mathrm{T}=11 \mathrm{~K}$ and held at this temperature under dynamic evacuation until an equilibrium pressure of $7.5 \cdot 10^{-2}$ Torr was achieved in the system. During this process, a fraction of the ${ }^{3} \mathrm{He}$ impurity was desorbed from the sample. The sample was then cooled back down to the lowest temperature, $2.1 \mathrm{~K}$, and the radial thermal expansion was measured again.

\section{RESULTS AND DISCUSSION}

The temperature dependence of the radial thermal expansion coefficient $\alpha_{r}$ of the ${ }^{3} \mathrm{He}-\mathrm{SWNT}$ system is shown in Fig. 17. Solid circles represent $\alpha_{r}$ of the sample with the initial ${ }^{3} \mathrm{He}$ concentration $9.4 \mathrm{~mol}$. \%, empty circles data for the same sample after partial removal of the ${ }^{3} \mathrm{He}$ impurity by heating at $11 \mathrm{~K}$. The inset in Fig. 1 1 a shows the low-temperature data for the partially evacuated sample on an expanded scale to enable a comparison with earlier studies, while Fig. 1b is shown on an intermediate scale for further comparisons with the saturated sample.

It is obvious from the Figure that saturating SWNT bundles with ${ }^{3} \mathrm{He}$ causes a dramatic increase in the magnitude of the negative thermal expansion in the interval 2.1-7 K. The largest negative $\alpha_{r}$ in the $9.4 \%{ }^{3} \mathrm{He}-\mathrm{CNT}$ solution exceeds those of pure CNTs and ${ }^{4} \mathrm{He}$-saturated CNTs by three and two orders of magnitude, respectively. As in the case of the ${ }^{4} \mathrm{He}-\mathrm{SWNT}$ solution, the negative contribution to the thermal expansion of the ${ }^{3} \mathrm{He}-\mathrm{SWNT}$ system is most likely due to a process of spatial redistribution of the ${ }^{3} \mathrm{He}$ atoms by tunneling at the surface and inside SWNT bundles. The isotope effect occurs

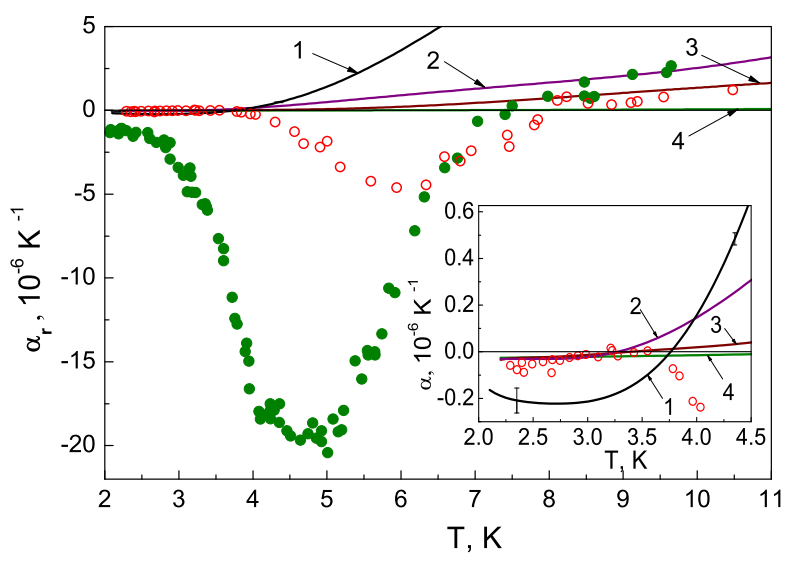

a)

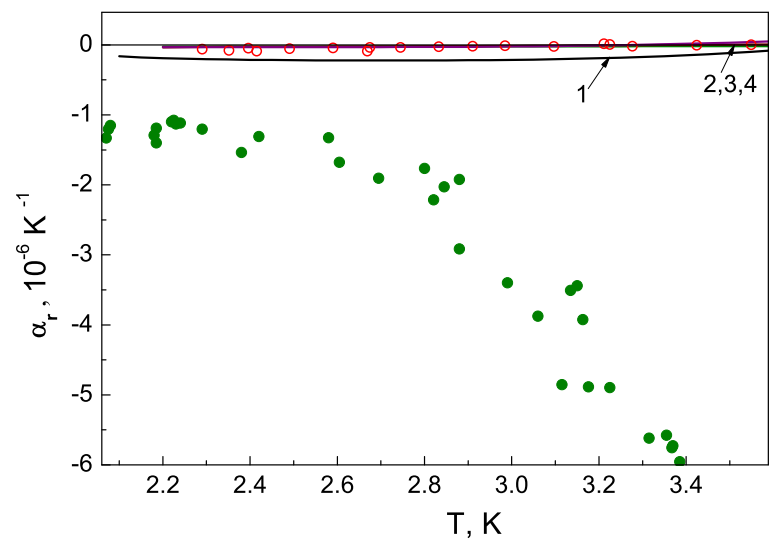

b)

FIG. 1. The radial thermal expansion coefficient $\alpha_{r}$ as function of temperature for SWNT bundles saturated with different gases. ("b" is a blowup of "a")

Symbols show data from the present study: $\bullet-{ }^{3} \mathrm{He}-\mathrm{SWNT}$, molar ${ }^{3} \mathrm{He}$ concentration $9.4 \%$; ० $-{ }^{3} \mathrm{He}-\mathrm{SWNT}$ after partial removal of the ${ }^{3} \mathrm{He}$ impurity at $\mathrm{T}=11 \mathrm{~K}$. Full lines show data from earlier work: $1 .{ }^{4} \mathrm{He}-\mathrm{SWNT}$, molar ${ }^{4} \mathrm{He}$ concentration $9.4 \%^{7}, 2 . \mathrm{H}_{2}-\mathrm{SWNT}^{5}, 3$. Xe-SWNT ${ }^{4}$, and 4 . data for pure $\mathrm{SWNTs}^{12}$.

because of the larger tunneling probability of the ${ }^{3} \mathrm{He}$ atoms, which have a smaller mass than the ${ }^{4} \mathrm{He}$ atoms, all other things being equal.

The temperature regions for the strong Schottky-like anomalies observed in the thermal expansion coefficients for the ${ }^{3} \mathrm{He}-\mathrm{SWNT}$ and ${ }^{4} \mathrm{He}-\mathrm{SWNT}$ solutions (similar anomailes have been predicted in specific heat of lowdensity He gas adsorbed in carbon nanotube bundles $\underline{14}$ ) suggest rather low energy barriers impending the motion of the He atoms in SWNT bundles. Strzhemechny and Legchenkova ${ }^{15}$ have used the potential curves ${ }^{16}$ for a helium atom interacting with the outer surface of a singlewall carbon nanotube in order to evaluate the tunneling probability of different He isotopes along the nanotube. They showed that in this direction a ${ }^{4} \mathrm{He}$ atom propagates 
in an energy band approximately $10.1 \mathrm{~K}$ wide. The respective band width for ${ }^{3} \mathrm{He}$ is $13.4 \mathrm{~K}$. These values are quite consistent with the results of this study.

After a partial ${ }^{3} \mathrm{He}$ desorption from the sample the negative contribution of the impurity to the thermal expansion decreases and shifts towards higher temperatures (Fig. 1a). The reason may be as follows. There are several kinds of sites where He atoms can reside in SWNT bundles, and the resulting total tunneling contribution to the thermal expansion is a sum of contributions made by various types of tunneling motion. On desorption, the He atoms leave first the sites with a comparatively low energy for the bond between the He atom and the C framework. As a consequence, the role of different types of tunnel motion changes, which affects the temperature dependence of the resulting tunneling contribution to the thermal expansion.

The authors are indebted to M. A. Strzhemechny for fruitful discussions and to the National Academy of Sciences of Ukraine for the financial support of the study (Program "Fundamental problems of nanostructural systems, nanomaterials, nanotechnologies", Project "The quantum phenomena in nanosystems and nanomaterials at low temperatures").
* dolbin@ilt.kharkov.ua http://www.dolbin.org.ua/

1 S. Iijima, Nature 354, 56 (1991).

2 M. W. Cole, V. H. Crespi, G. Stan, C. Ebner, J. M. Hartman, S. Moroni, and M. Boninsegni, Phys. Rev. Letters 84, 3883 (2000).

3 J. V. Pearce, M. A. Adams, O. E. Vilches, M. R. Johnson, and H. R. Glyde, Phys Rev. Lett. 95, 185302 (2005).

4 A. V. Dolbin, V. B. Eselson, V. G. Gavrilko, V. G. Manzhelii, N. A. Vinnikov, S. N. Popov, N. I. Danilenko, and B. Sundqvist, Fiz. Nizk. Temp. 35, 613 (2009), [Low Temp. Phys. 35, 484 (2009)].

5 A. V. Dolbin, V. B. Esel'son, V. G. Gavrilko, V. G. Manzhelii, S. N. Popov, N. A. Vinnikov, and B. Sundqvist, Fiz. Nizk. Temp. 35, 1209 (2009).

${ }^{6}$ A. V. Dolbin, V. B. Esel'son, V. G. Gavrilko, V. G. Manzhelii, S. N. Popov, N. A. Vinnikov, and B. Sundqvist, Fiz. Nizk. Temp. 36, 465 (2010).

7 A. V. Dolbin, V. B. Esel'son, V. G. Gavrilko, V. G. Manzhelii, N. A. Vinnikov, S. N. Popov, and B. Sundqvist, Fiz. Nizk. Temp. 36, 797 (2010).

8 Y. A. Freiman, Fiz. Nizk. Temp. 9, 335 (1983), [Sov. Low
Temp. Phys. 9, 335 (1983)].

${ }^{9}$ V. Narayanamurti and R. O. Pohl, Rev. Mod. Phys. 42, 201 (1970).

10 A. N. Aleksandrovskii, V. B. Esel'son, V. G. Manzhelii, B. G. Udovidchenko, A. V. Soldatov, and B. Sundqvist, Fiz. Nizk. Temp. 23, 1256 (1997).

11 N. Bendiab, R. Almairac, J.-L. Sauvajol, and S. Rols, J. of Appl. Phys. 93, 1769 (2002).

12 A. V. Dolbin, V. B. Eselson, V. G. Gavrilko, V. G. Manzhelii, N. A. Vinnikov, S. N. Popov, and B. Sundqvist, Fiz. Nizk. Temp. 34, 860 (2008), [Low Temp. Phys. 34, 678 (2008)].

13 R. H. Sherman, S. G. Sydoriak, and T. R. Roberts, J. Res. Nat. Bureau Stand. 68A, 579 (1964).

14 A. Šiber and H. Buljan, Phys. Rev. B 66, 075415 (2002).

15 M. A. Strzhemechny and I. V. Legchenkova, (2011), private communication, the article is to be submitted to Fiz. Nizk. Temp.

16 L. Firlej and B. Kuchta, Colloids and Surfaces A: Physicochem. Eng. Aspects 241, 149 (2004). 